\title{
Energy Management And Safety Trends In Mechanical Analogous Thermal Model of Building System
}

\author{
Vishnu Narayanan and Geetha Mani
}

\begin{abstract}
With the advent of increase in scientific gadgets and technological intervention in almost all the working sectors, utilization of energy has become a prime mandate in deciding the economy and growth. Moreover with urbanization at its peak, sophisticated building technology is also developing which is now one of the major consumers of the generated energy, especially for its Heating Ventilation and Air Conditioning (HVAC) systems. This current scenario has urged to find a holistic way to improve energy management in buildings for a sustainable future resulting in Building Energy Management System (BEMS).This paper aims at developing an energy management platform for the building model; where-in a mechanical analogous thermal model of a building system is framed. Model Predictive Control (MPC) algorithm is employed with the integration of Building Management System (BMS), wherein the simulation was conducted to ensure the vigour of MPC to be employed as a supervisory control strategy. Safety aspects of this energy management methodology along with emerging trends is also been discussed in detail in this paper.
\end{abstract}

Keywords: - BMS, Energy Management System, MPC, HVAC.

\section{INTRODUCTION}

Even though the conscious regarding building automation and proper control of HVAC systems in a building environment began to evolve in the late 1970s, it is only after the reckless load shedding and power quality problems around early 1990s energy management in buildings became a demanding factor in socio-economic growth [1]. With the development of technologies associated to automation and communication, BEMS has taken a long route since its emergence. Recently an important factor that promoted the development of building automation is related with energy issues. The high frequency of energetic crisis and the corresponding pressure on energy prices motivates the development of energy intelligent management systems [2]. Moreover, constant employment of HVAC systems would radiate green-house gas and that would in-turn contribute for the global-warming. This has been depicted as a serious issue, as energy management not only affects social and economical trends, but also has a major impact over environmental changes and its impact over future generations. Hence proper management of energy in building environment, as it is a major consumer of energy becomes mandatory. A building, is assessed by parameters such as ventilation rate, degree of insulation, occupancy rate, location of thermal capacity, glazing type, amount of HVAC provision, level of control and nature of fuel, to

Revised Manuscript Received on October 15, 2019.

${ }^{1}$ Vishnu Narayanan, Sr.Engineer, Robert Bosch, Coimbatore, Tamilnadu, India. (Email: vishnu92narayanan@gmail.com)

${ }^{2}$ Geetha Mani, Associate Professor, School of Electrical Engineering, Vellore Institute of Technology, Vellore, Tamilnadu, India.

(Email: geethamr@gmail.com) point a few. Thus even a building with relatively less number of parameters will pretend to be very complex in design. Hence, concentrating on thermal characteristics and contributors of temperature variation inside and around a building can be taken into account for designing the building model $[3,5]$. By effective control of thermal model, energy can be saved to a large extent with minimal damage to environment. Thermal design of a building system is very critical as it involves various parameters and implicit interactions between the parameters. It accounts for several thermodynamic domains such as air dynamics, radiation exchange, extent of moisture, electrical power flow, heat source, sinks etc--each one of which would interact with the others in a non-trivial fashion. For example, the simple act of adjusting the position of a window shading device will have cascading effects on adjacent wall temperature, thermal gradient, Air-Conditioner temperature requirement, heating and electricity demand [6]. Thus a proper thermal model is required which can be of either electrical analogous model or mechanical analogous model. Electrical thermal model would comprise of resistances and capacitances which would act as thermal resistor and heat storage devices; on the other hand, mechanical thermal model will hold conductive and convective heat transfers with associated coefficients, heat flow direction, thermal mass of wall, window, roof etc [8]. Upon formulating the thermal model of the building, proper choice of controller and corresponding actuator becomes critical. Starting from bangbang controller, there are numerous numbers of controllers available that could cater the requirement with various degree of precision. Conventional PID controller can be employed on elucidating the first order approximation of the building system [7]. It should be taken into account that upon approximating the thermal model as a first order system, the model would be well behaved for various inputs, but it can't be implemented in real time as it would be devoid of major interactions between various model parameters. Considering the fact that PID cannot handle constraints and operate only on delicate systems, standalone PID cannot be implemented for this system. Combination of two controllers would result in a better output; say PID can be used along with any other adaptive mechanism or fuzzy based controller to improve the output characteristics. Various theories and implementations suggest that Model Predictive Controller (MPC) gives better output satisfying the optimization problem within the stipulated constraints $[9,10]$. But, it is to be ensured that 
MPC can be used only at supervisory level and one more conventional or modern application based controller must be included in-order to make the total control system as a practical one. Even the commercially available Thermally Activated Building Systems (TABS) referring to heating and cooling transmission systems, which takes advantage of large surface areas and integrates the building structure, employs the predictive mechanisms to radiate the heater or inject the coolant via hydronic tubes [11]. TABS are proven to be economically viable and impart right control action. The success of TABS technology in HVAC control in a building system is due to two major factors, namely hydronic circuit topology and the employment of MPC for control strategy $[12,13]$. Thus utilizing MPC with the right constraints and optimizing function would result in a formidable control.

Apart from conventional way of modelling and control, MLE+, a tool for integrated design and deployment of energy efficient building controls can be employed. MLE+ software can be coupled with MATLAB platform through BACNet communication link so that the design which is being implemented in MLE+ can be incorporated in MATLAB simulation software [14]. Building Energy Performance Simulation (BEPS) tools (e.g. EnergyPlus, TRNSYS, ESP-r, etc.), which are typically used for soft modelling of the building structure is the major component of MLE+. These tools consists of various complex calculations, switches, non-linearity and iterative tasks that make their outcome in online optimization prohibitive as the resulting models are in an implicit form. As the method using MLE+ involves SI, the model obtained would entirely rely on the data collected. If the data does not have considerable likelihood, then it would result in an approximate modelling. Hence this method is not preferred much; however research works are going on over utilizing MLE+ effectively for HVAC control. Building model design can also be done using Google SketchUp software, where-in the Google earth is used to take snapshot of the real building structure whose model is to be designed. By employing the features available at SketchUp software terminal, building with necessary thermal features like doors, windows etc. can be framed and this model can be exported to MLE+ co-simulation software interface called Building Controls Virtual Test Bed (BCVTB), using which control action can be implemented over this model using MATLAB [16]. Authors suggests this method if real-time snapshot can be taken with very high clarity and accuracy.

Commercially available smart thermostats and other HVAC control devices employs MPC algorithm to efficiently accommodate with the comfort laws imposed by the customers within the constraints to envisage BEMS. Actuators are usually thermostats, heater or coolant injecting pump, whose input is varied using the MPC controller's output and the output from the actuator is fed into the building where-in temperature has to be controlled with optimized energy efficiency. Temperature sensors are used in real time to monitor the current temperature values and the values are fed back to MPC to control the temperature forming a closed loop.

The complete system predicts the indoor framework conditions of a specific building considered, and selects the most appropriate proceedings so as to attain the set point and lead to the indoor environmental quality by mitigating energy cost. The performance of the illustrated model design and the outcomes due to the presence of predictive controller is analyzed by simulating the whole system using MATLAB Simulink tool. A number of important characteristics pertaining to various real world conditions like constrained execution, cost-performance trade-off, lifetime and safety are explicated elaborately with simulation results as the evidence.

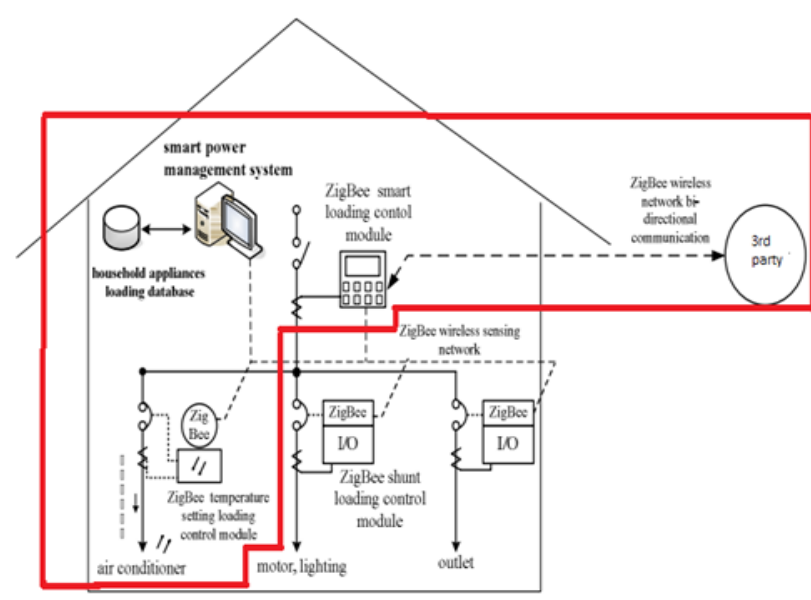

Fig. 1.Building Heating System with Smart Communication Network

Major outcome of this paper would be providing an insight as to why and how energy management can be effectively performed with the main concentration over the control of HVAC systems, which forms the prime consumer of overall energy supplied to the building system. Amidst satisfying the said goal, with the effective completion of the paper, it would be an eye opener for environment friendly or sustainable future, making all the existing building systems into zero energy smart structures. In practical sense, communication also plays a vital role in claiming this work to be a fit for market product work. ZigBee technology can be envisaged along with the right control strategy in-order to rightly frame the communication platform as in Fig.1.Eventhough this RF technology has been used partly at various junctures possible in home automation with respect to energy, still there is a large room for the integration so that a net effective management system will be the outcome.

\section{SYSTEM ARCHITECTURE}

Thermal system in general will comprise of various components that govern the heat transfer rate, temperature variation, heat sources, sinks and other thermal specifications. All the specifications will hold few of the basic physical laws which form the first principles of thermal system. Few of these laws are illustrated as follows [3]. 
Fourier's law of Conduction states that heat flux is directly proportional to temperature gradient $\left(\frac{d T}{d x}\right)$

$$
q=-k\left(\frac{d T}{d x}\right)
$$

Where $k$ corresponds to thermal conductivity and $x$ represents isotropic thickness. In most cases $x$ is approximated as a constant. For steady state heat transfer $k$ is also a constant, hence the above equation can be integrated as,

$$
\begin{aligned}
& \int_{x_{1}}^{x_{2}} q d x=\int_{T_{1}}^{T_{2}}-k d t \\
& \dot{q}\left(x_{2}-x_{1}\right)=-k\left(T_{2}-T_{1}\right) \\
& \dot{q}=-k \frac{\left(T_{2}-T_{1}\right)}{\left(x_{2}-x_{1}\right)}=-\frac{\left(T_{2}-T_{1}\right)}{R}
\end{aligned}
$$

Where, $R$ represents thermal resistance.

$$
R=\frac{\left(x_{2}-x_{1}\right)}{k}
$$

Moreover, Faraday's law also suggests that,

$$
\frac{\partial^{2} T(x, t)}{\partial x^{2}}=\frac{1}{\alpha} \frac{\partial T(x, t)}{\partial t}
$$

Where, $\alpha$ corresponds to diffusive area.

Newton's law of convection is one another postulate that elucidate thermal dynamics. Newton proved that heat flux due to convection depends relatively on the difference in air temperature $\left(T_{\text {air }}\right)$ and surface temperature $\left(T_{w}\right)$.

$$
q=h_{c}\left(T_{a i r}-T_{w}\right)
$$

Where $h_{c}$ corresponds to convective heat transfer coefficient which is related to flow state and natural convection of air flow.

Convection can be illustrated as, solar energy will intend to rise the surface temperature, which will in turn increase the rate of release of energy to the neighbourhood air by the phenomenon of natural convection. Considering a building, convection is usually and largely influenced by wind forces.

A practical thermal system will predominantly composed of conduction and convection heat transfers, but there exists another mode of heat flow, called as radiation. A perfect example of radiation is the way how sun's heat is transmitted throughout the earth surface. StefanBoltzmann's law gives the following lemma.

$$
q=\sigma \varepsilon_{1} \varepsilon_{2}\left(T_{1}^{4}-T_{2}^{4}\right)
$$

$\sigma$ and $\varepsilon$ correspond to Stefan-Boltzmann constant and surface radiance respectively.

Other important relations that contribute to the characteristics of a thermal system are listed below [5].

Rate of heat storage within a region is given by,

$$
q=(V) \rho c_{p}[T(t+\delta t)-T(t)]
$$

Here Vrepresents the region volume. $\rho$ directs to characteristic density of the considered thermal region and $c_{p}$ is the specific heat capacity.
Heat capacity is framed through,

$C=\rho c_{p}$

Thermal mass $=T_{m}=V \rho c_{p}$

Above equations would give a clear picture on how the considered thermal system will react upon varying temperature conditions.Typical Heating/Cooling schematic diagram is elucidated in Fig.2.

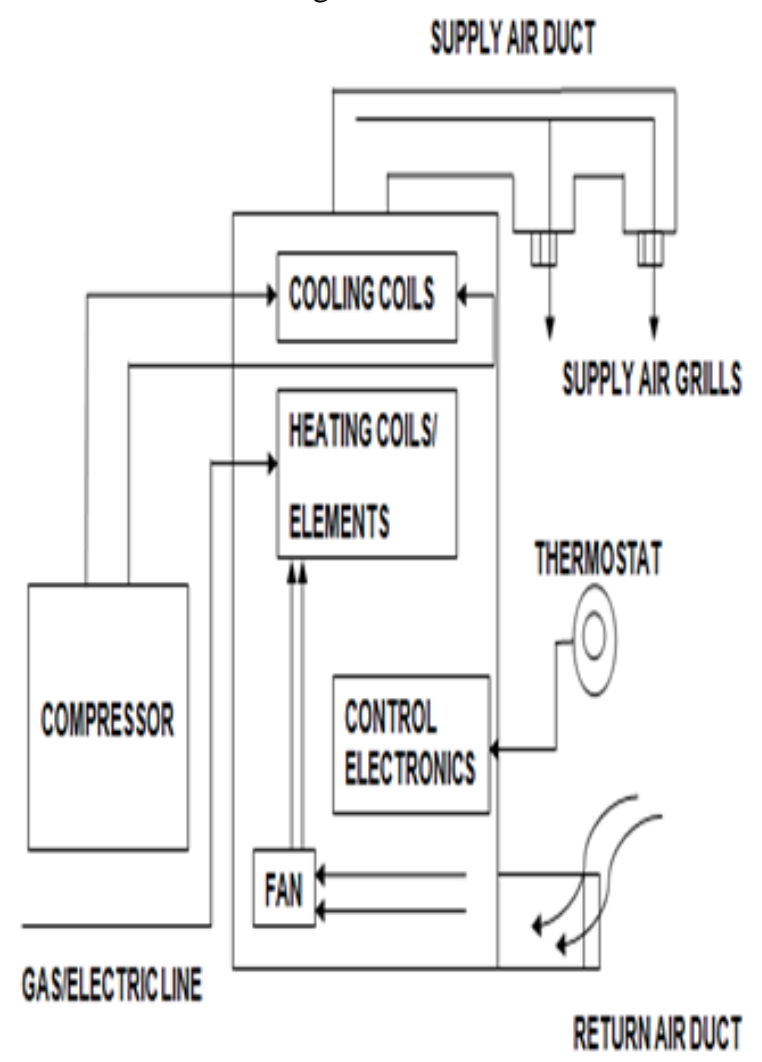

Fig. 2. Schematic Diagram - Thermal System comprising of HVAC components

From theFig. 2, it is very much obvious that each and every component in the HVAC system demand enormous amount of energy for its operation. Heating can be done either by passing gas/fluid through hydronic tubes that impart heat energy or by circulating electric current; again, energy is demanded for pumping in the case of former and generating electricity in the case of latter. For the process of cooling, compressor has to be run continuously with a very high pressure created artificially, which demands a lot of energy. Sucking and evacuating the excess air also requires energy for its operation. Thermostat instructs on how and when to vary the thermal coefficients in-order to meet the required demands [2]. This is for a single HVAC system. Similarly there exists innumerable number of such systems which draw energy in leaps and bounds. On the other hand, the coolant employed, which is invariably ChloroFluoroCarbons (CFCs) which destroy the 
environment by depleting ozone layer and letting Ultra Violet (UV) radiations to spread over the earth. Such a simple HVAC system can have such a massive impact.

The International Energy Conservation Code (IECC) gives standards and codesthat HVAC systems often use in developing building energy monitoring scale at the internal level. Most building energy management systems are operated using specially designed software programs that continuously monitors the effectiveness of installed HVAC systems. These programs are run using a normal computer, and are capable of elucidating feedback on system working and energy consumption.The monitoring and trending capabilities of EMS are powerful tools for enhancing HVAC system performance and for reducing energy use, but most system operators simply do not have enough time to fully investigate these vast resources. Those who are responsible for EMS improvement and upgrade, or purchase are not able to study their facility's exact EMS needs themselves; they may rely on vendors to provide specifications-not receiving the optimal frame for their building. If the EMS system is effectively integrated for a Building system, then it results in an effective BMS. Modelling of the building is a topic of concern while developing efficient building energy management, especially in case of TABS. Electrical analogy is usually employed to come out with a building model. Electrical counter for the building components would consist of purely resistors and capacitors, where suffix ', corresponds to electrical analogous components. Capacitors,its nature suggests it stores the heat energy and the resistors mitigate the heat flow through various surfaces. Resistance offered would vary depending on whether the heat flow transfer is conduction, convection or radiation; whereas capacitors can hold only a predetermined fixed amount of heat irrespective of the mode of heat transfer [12]. The flow of heat in and around the building structure is associated with the flow of current in an electrical circuit. Modelling is done as though these electrical components are lumped and not distributed for easy mathematical access and to get hold of the interaction between various building components [8]. Numerical values for the resistors and capacitors are provided depending on the size and orientation of the room; also by taking into account the surrounding conditions of the building. One such electrical analogous thermal model of a room is as shown in Fig. 3.

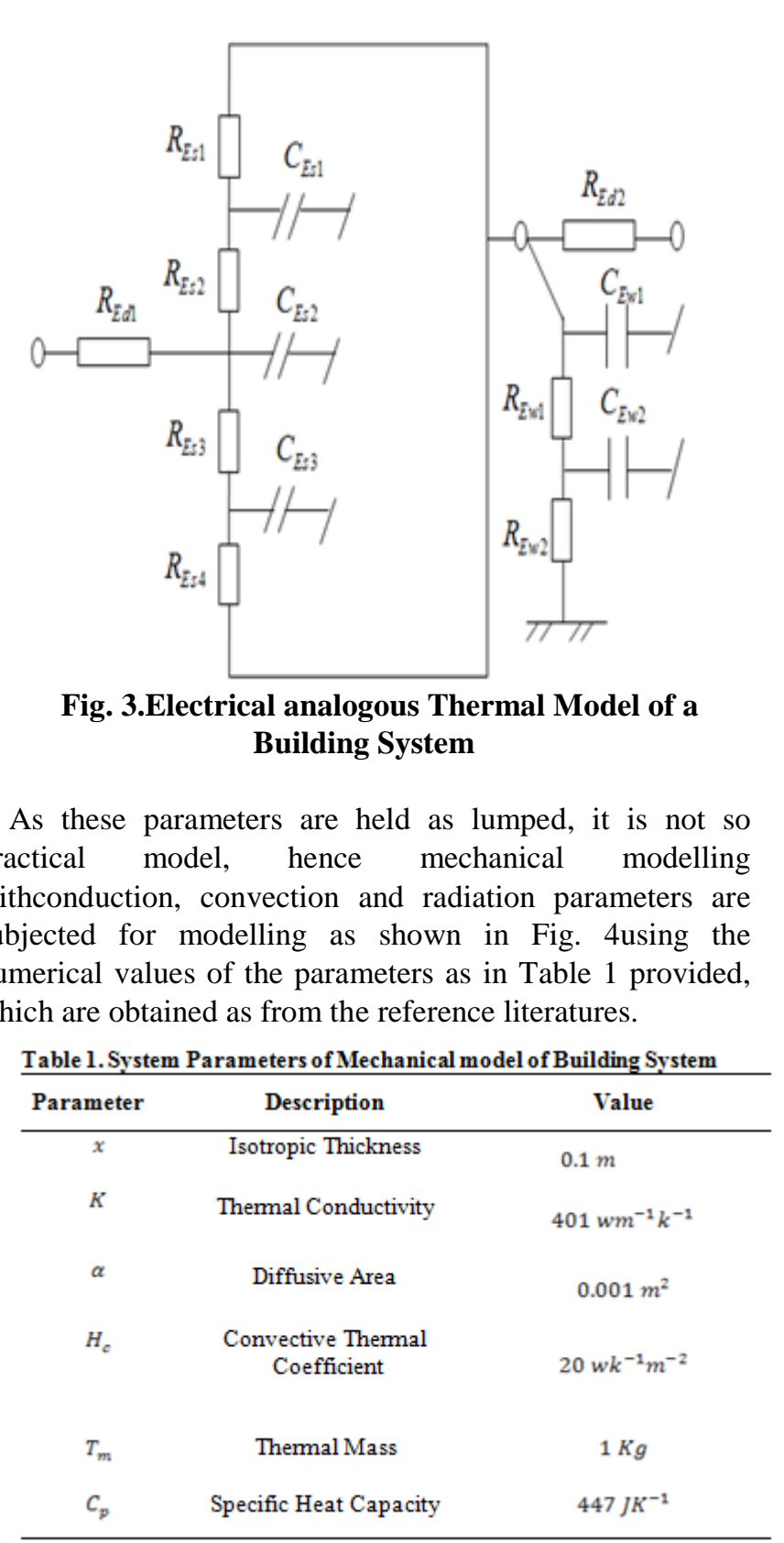




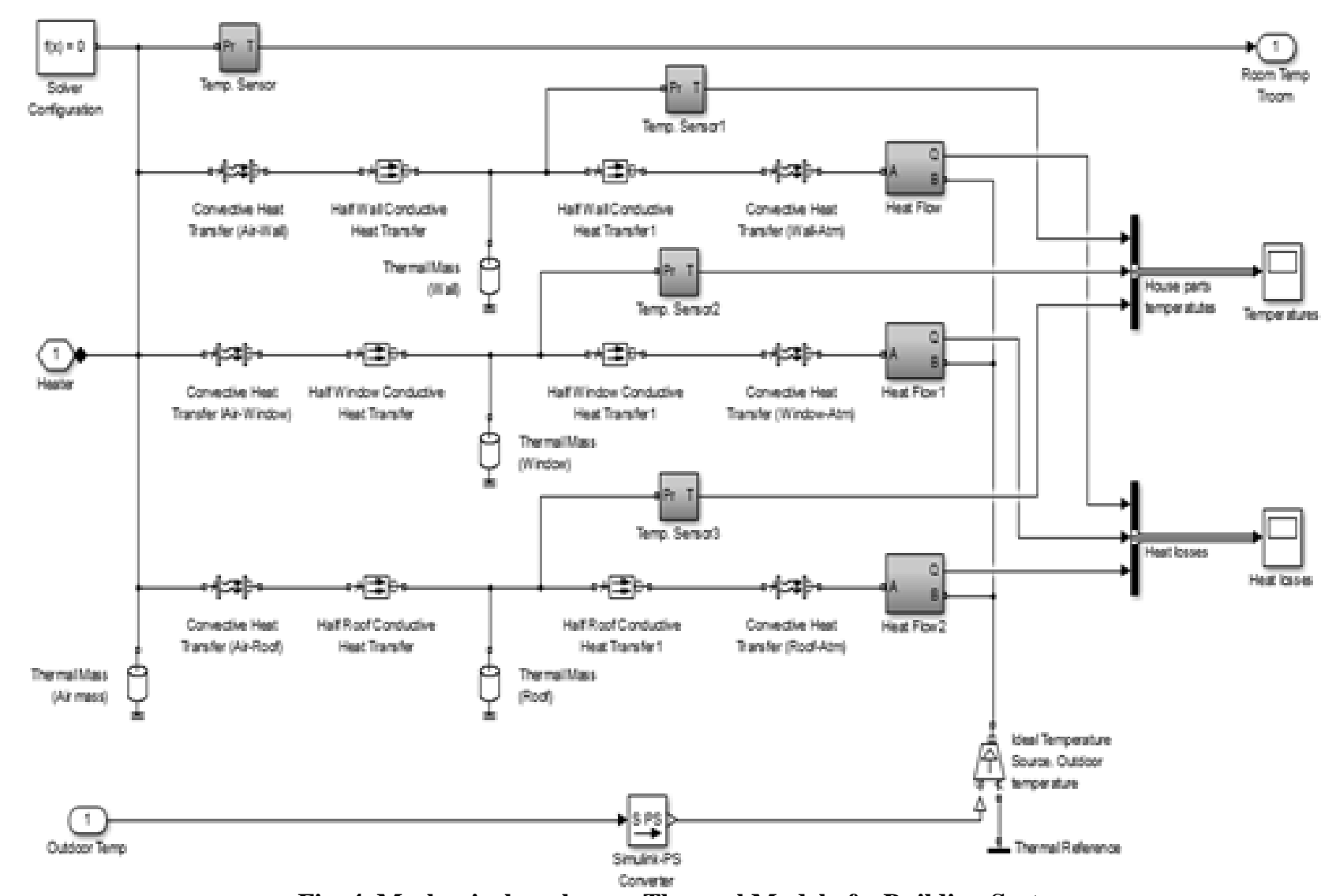

Fig. 4. Mechanical analogous Thermal Model of a Building System

Mechanical system obtained here is for a single room building system, it can also be extended for multiple rooms by appropriately altering parameters of Table 1 .

\section{CLOSED-LOOP MODELING}

With the main objective being formulation of the building control as an optimization problem, the building physics are formulated in a mathematical model that is used for the prediction of the future building behaviour according to the selected operation strategy. Now, the aim is to develop a control strategy, that reduces the energy consumption ( operational costs) while guaranteeing that all comfort needs are met; for which MPC controller is chosen. The key principle of MPC used for building energy control is the efficient use of the thermal storage or thermal mass of a building. MPC is not the stand-alone technique, but an effective one that can be used for optimal building control. Literature shows that energy saving capability of this method reaches almost $40 \%$ compared to classical control strategies based on the particular building type [9].

In-order to design a MPC, input and output parameters of the controller is are needed to be segregated and identified. Time-varying parameters (i.e. the energy price, the comfort criteria, as well as predictions of weather and occupancy) are inputs to the MPC controller; whereas the manipulated variable that gives input to the actuator controlling the temperature forms the output. It is evident that design and modelling effort consists of identifying a dynamic model of the building, constraints of the control problem and a cost function that encapsulates the requiredbehaviour. At each sampling period, these components are collaborated and converted into an optimization problem based on the MPC framework chosen. This optimization happens on the specific constant time interval, which is called prediction horizon with the length specific for the control problem solved. Specifically the cost function of a HVAC system is equivalent to energy tapped and penalty for few comfort requirement violation. The major point, which really varies from other control strategies is just that, in MPC given constraints are manipulated into control strategy, hence it predicts potential future saturations and picks up an effective control trajectory, which minimizes the cost function and without violating the constraints all at once. Fig. 5 depicts the application of MPC over a building model with its input and output parameters.[17]

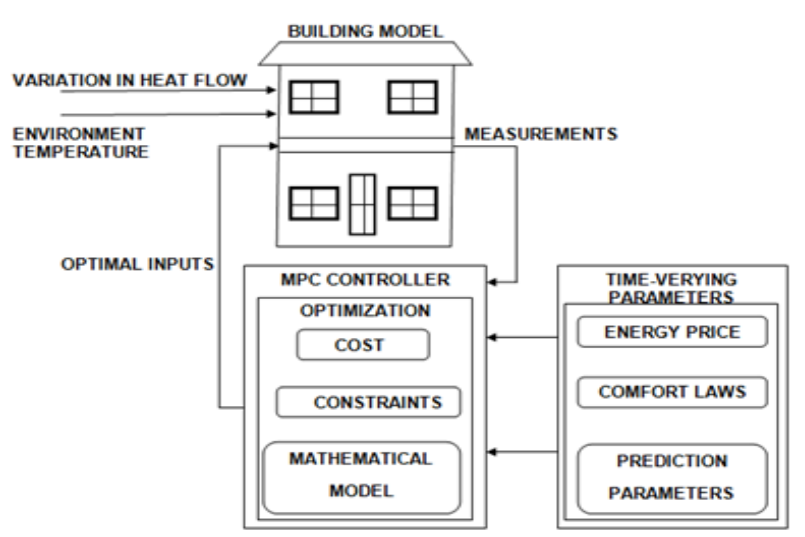

Fig. 5. Closed Loop Control of Building Model using MPC 


\subsection{Cost Function}

A cost function is usually an optimization problem which could be solved using any of the optimization solvers pertaining to the cost function.

The cost function generally serves two purposes, namely;

- STABILITY - It is common to choose the structure of the cost function such that the optimal cost forms a Lyapunov function for the closed loop system, and hence will guarantee stability

- PERFORMANCE TARGET - The cost is generally, but not always, used to specify a preference for one behaviour over another, e.g., maximum comfort or minimum energy

$$
J=\min \sum_{k}^{N-1}\left(y_{k}-y_{r, k}\right)^{\tau} Q\left(y_{k}-y_{r, k}\right)+R U_{k}
$$

Here, $y_{k}$ and $y_{r, k}$ depict measured output and the reference output values, with $U_{k}$ being control input. $Q$ and $R$ represents weighing matrices of appropriate size. The trade-off between energy consumption and precision of reference tracking and is expressed by proportion of the matrices $Q$ and $R$.

\subsection{Dynamics and Constraints}

Constraints are characterized input equality and inequality constraints. If the input or output limit is stipulated to be some numerical equivalent, then the constraint is said to be equality constraint; on the other hand if the input or output can vary between a range of values, then it is termed to be inequality constraint. Change in input is not taken as a constrained value for this application, but in general it can also be given with a constrained limit. Inequality constraint pattern is chosen for the input-output pair.

Input constraint:

$$
U_{\min } \leq U \leq U_{\max }
$$

Output constraint:

$$
y_{\min } \leq y \leq y_{\max }
$$

Minimum and maximum values of input and output can be set based upon the system and the physical significance and limitation of the actual input and output signals.

\subsection{Closed Loop Control}

The very same closed loop control of the process that is the building system can be brought out using MATLAB Simulink tool. Dedicated MPC block is present, where-in theappropriate parameters chosen as depicted in the previous sections of this chapter is fed. It can also be fed through a separate MATLAB code, which integrates the numerical values provided for MPC from workspace to the MPC block. Mechanical analogous thermal model of the building system as in Fig. 5 is made as a subsystem and renamed as 'House', to increase the readability and understanding ability. Actuator is chosen as a thermostat as mentioned before, which has been depicted as 'Heater'. The complete MATLAB setup consisting of all these parameters is shown in Fig. 6.

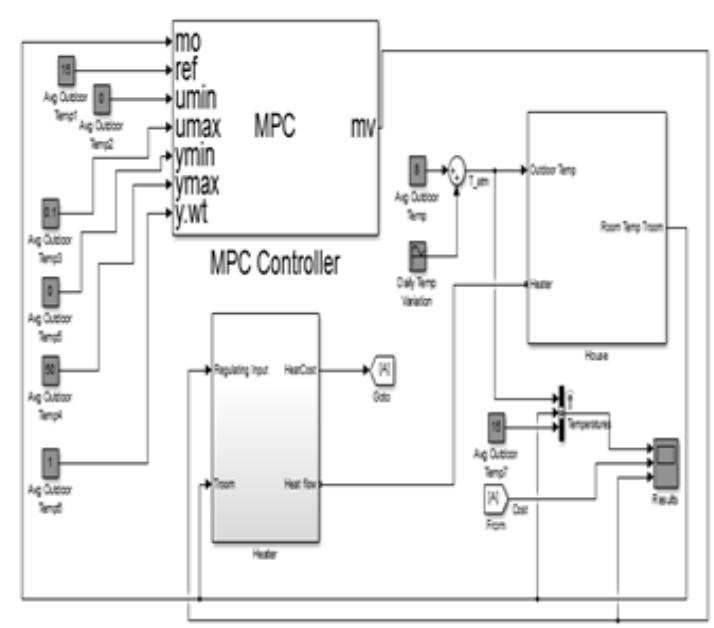

Fig. 6. Closed Loop Control of Thermal Model of Building System

Table 2 is employed in simulating the model designed. All the numerical values are obtained from various reliable references. Various outputs are attained with different test conditions, concentrating both on total energy consumption as well as on the expenditure. Apart from these two explicit criteria, the control effort is also analyzed using the resultant graphs obtained through simulink. It was inferred that control effort is also one major area wherein the optimization constraint can be set.

The system description is usually depicted in state space representation and upon applying corresponding numerical values it would result in system and input matrix.

$$
\begin{aligned}
& \dot{X}=A X+B \\
& Y=C
\end{aligned}
$$

Where, is the state, is the control input and corresponds to output. The state space model

\section{Table 2.Controller Parameters and Constraints}

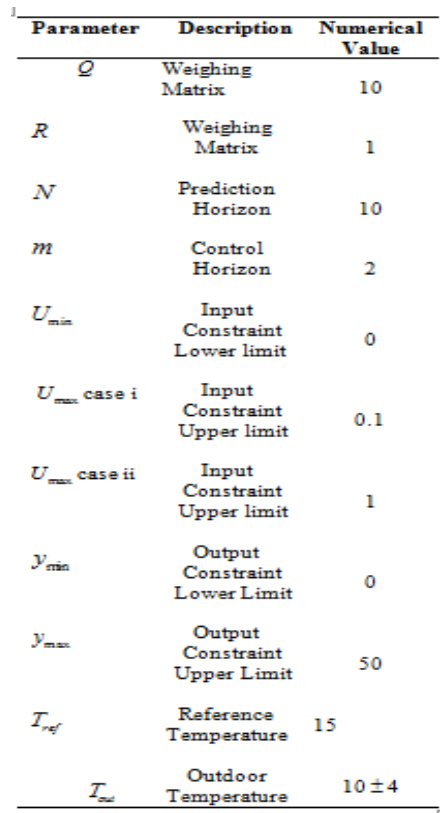


of the system is obtained through linearization technique.These matrices are employed along with various test conditions in-order to rightly analyze the system and the controller.

\section{RESULTS AND DISCUSSION}

\subsection{Optimized Temperature Control}

Temperature control results are depicted in the forthcoming sections:

\section{A. Reference Tracking}

External temperature and room temperature are plotted along with the reference temperature.

$$
x_{0}=20
$$

Where corresponds to initial room temperature set in degree Celsius.

$$
T_{r e f}=15
$$

Reference temperature is set with 15 degree Celsius.

$$
T_{\text {out }}=10 \pm 4
$$

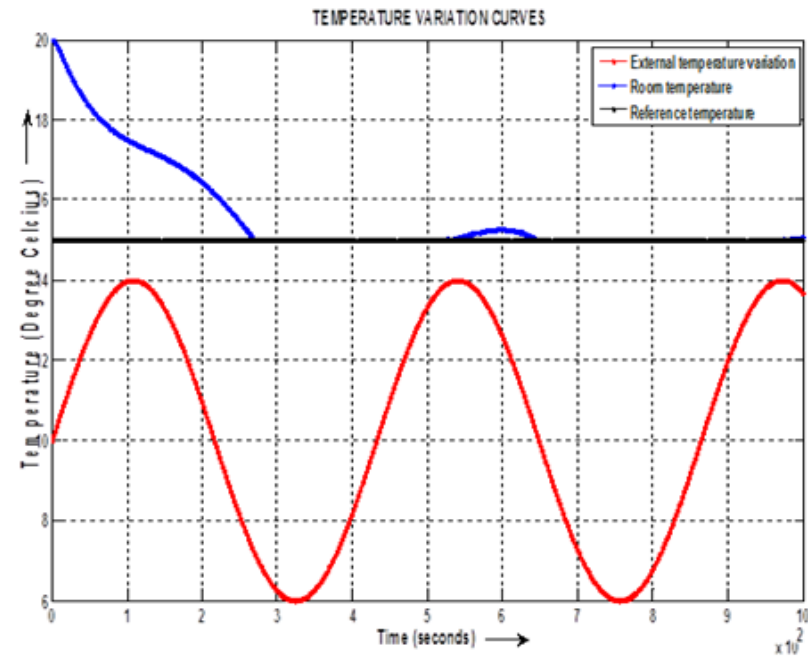

Fig.7. Set-point Tracking amidst the presence of External Temperature variation

It is obvious that environmental temperature won't vary drastically. The sinusoidal temperature variation is natural as temperature increases during day and reaches negative maximum during night; this repeats to give a sinusoidal temperature variation. But the temperature gradient, i.e. difference between the old and new temperature is very minimal. Almost 8 degree Celsius variation is provided which is unnatural as in Fig.7. But this variation is purposefully given as in Fig.6 in-order to check the controller's dominance. Even amidst this variation and the continuous effect of windows (which is usually kept closed during the operation of HVAC systems) the room temperature tracks the desired set point within few hours, which doesn't happen with the ordinary controllers. There are no undesirable effects in the tracking trajectory, which is very smooth and doesn't seem to be sluggish on keeping in mind the extent of sluggishness that is inherent in a thermal process.

\section{B.Optimization}

Common cost calculation cannot be deployed to prove that the control input is optimized; yet a generalization can be done. By considering the fact that whenever control input varies, cost is increased. Control effort in the case of altering temperature would involve increasing or decreasing the regulator of thermostat. Both these actions would result in increase of cost as there is a regulation over the supplied energy. The Fig.8 clearly implies that the variation of cost doesn't involve any drastic variation even-though the controller seems to act continuously indicating that controller action is such that the cost is minimized. Moreover, the cost curve is very smooth indicating lot of energy is saved.

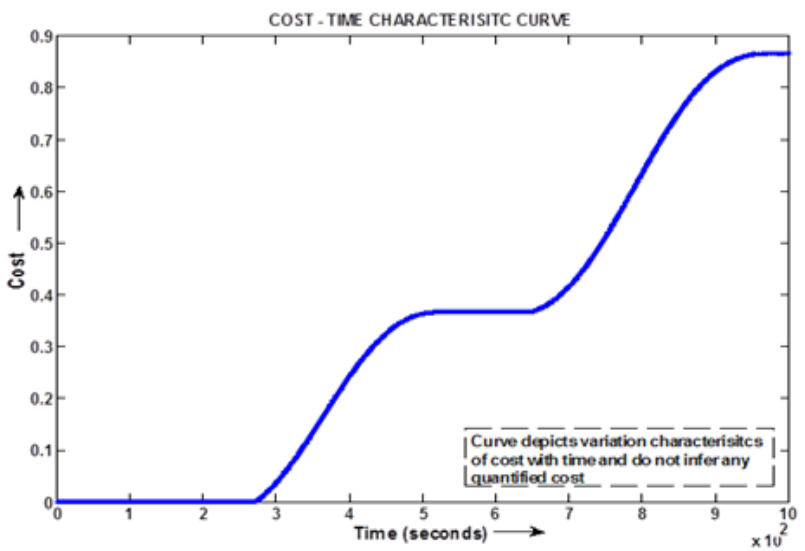

Fig.8. Optimization curve - variation of Cost with Time

C.Part-wise Temperature variation

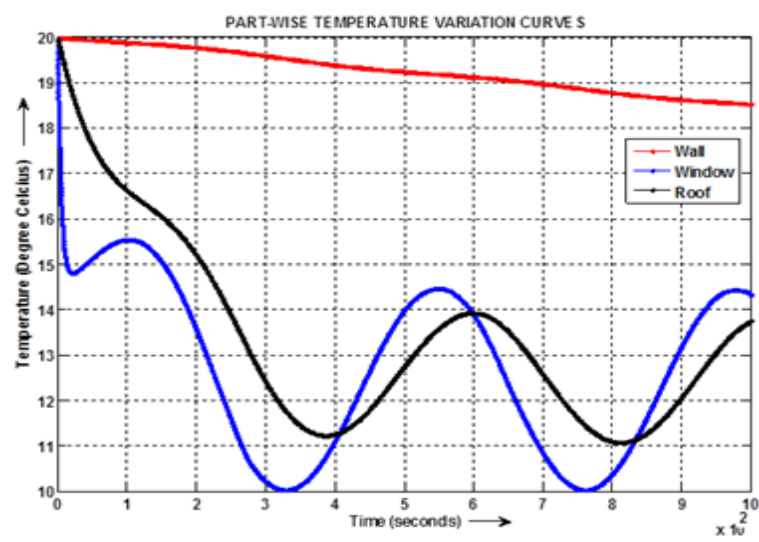

Fig.9.Part-wise Temperature variation curve of Thermal Building Model

Even-though the set point has been reached with minimal energy spent, it is mandatory to study the system considered in-order to have scope for future development. There are three components of room that are involved in modelling of mechanical analogous thermal model of building system; namely wall, window and roof. Now the task is to verify how the temperature in each of these components has varied in-order to bring out a total variation in the room temperature. This is said to be dissection of the paper, 
where-in intricacies that are not explicitly let-out are identified and analyzed. Variation of temperature in these three parts can be termed as part-wise temperature variation. Even-though controller gives optimal control signal to the actuator and even if actuator responds positively, there must exist a natural responder to the actuator's variations. To get hold of the right picture of both controller and the modelled building, this analysis is done. It is very clear from the Fig. 9 that wall has the property to retain the temperature. On the other hand, both roof and window aids in heat transfer continuously with a larger variations in the temperature. Between the roof and window, window still holds an upper hand in varying the temperature to a large extent with a very quick response time compared to the wall and roof. This gives us a fact that upon concentrating on the window for temperature control, the control action might have been more effective or a new ideology in control of thermal process can be discovered

\section{Table 3. Part-Wise Temperature Variation Range}

\begin{tabular}{cl}
\hline Part & Temperature variation \\
\hline Wall & $20 \leq T_{\text {well }} \leq 18.57$ \\
Roof & $20 \leq T_{\text {rod }} \leq 11.04$ \\
Window & $20 \leq T_{\text {wadew }} \leq 10$ \\
\hline
\end{tabular}

Table 3 gives the numerical values of the temperature variation of various parts of the building.

\subsection{Duty cycle of Controller and Safety}

It is mandatory to check how much is the controller burdened in-order to make to measured temperature reach to reference temperature. This aspect of analysis has a greater significance in determining how robust a controller must be and how much a controller can work on in-order to satisfy the requirement

This also gives a gist for the hardware design. Based upon the duty cycle or frequency with which the control signal is delivered, clock frequency, Random Access Memory (RAM) capacity and processor speed can be decided. Moreover other interfacing components like Analogue to Digital Converter (ADC) and Digital to Analogue Converter (DAC) can be chosen with proper bits and resolution to meet up with the controller speed and working capability. As almost every control algorithm in industry is implemented through a computer, selection of these interfacing components also plays a vital role

Every time the controller responds, that would result in the variation of manipulated variable. Amplitude-Frequency distribution plot of the manipulated variable would clearly give admissible idea over how the controller operates over time to meet the constraints as well as perform the job of elevating the room temperature to the set temperature.

Case i

$$
0 \leq U \leq 0.1
$$

From Fig.10, Manipulated variable is altered continuously making the controller to operate recklessly for several hours without any intermittent stop. If the input constraint is set for a very low range of values, with just 0.1 interval, then there is no other go for the controller but to act continuously in-order to track the set-point as well as optimize the input.

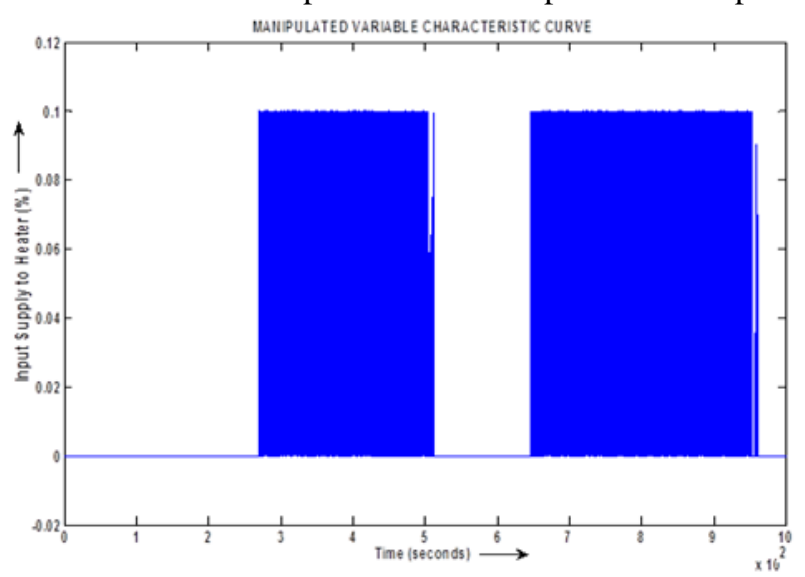

Fig.10. Duty cycle of Controller over Building Model (Case i)

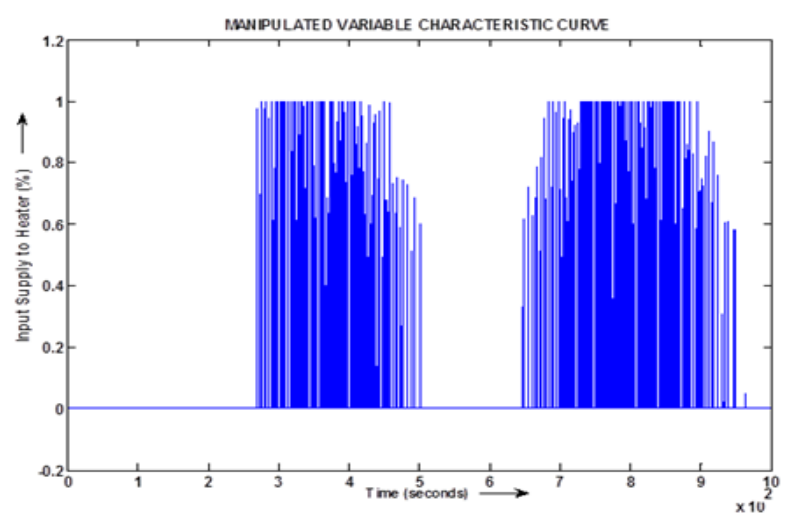

Fig.11.Duty cycle of Controller over Building Model (Case ii)

Case ii

$0 \leq U \leq 1$

It is obviously evident that the controller has rest duration at times between its operations in case of Fig.11, which is totally absent in Fig.10. The intermittent operation with a proper allocation of work-break period is very important on seeking a better life time for the controller. If controller's maximum operating capacity is utilized every second, then the life time of the controller decrease exponentially leading to controller failure. This can happen in real time, thus proper safety measures and redundant operation of controllers with effective back up is required. Even if the primary controller fails, the controller which is kept in shelf as a back-up can take up the job and continue from the juncture where the its predecessor left. This action of compensation during failure, adds avery vital contribution towards the entire control system and safety 


\section{CONCLUSION AND FUTURE SCOPE}

The system considered is by nature very sluggish and it was inferred from the closed loop operation of the system with the controller makes the system track the desired temperature in a trajectory that optimizes the energy spent, amidst the external temperature variation. Cost curves obtained from virtual experimentation implied that MPC critically contributes for energy optimization. The variation of temperature over different parts of the building suggested that window contributes for the majority of heat flow in a building, where-as the least is the wall

The nature of curve corresponding to the duty cycle of the controller instils the thought that even the operating time of the controllers can become a criterion in determining the performance of the controller. The curve also explains on how a redundant controller becomes critical for ensuring safety. Overall, the obtained results show greater performance of the controller in all aspects towards Building Management System (BMS).

Even though building automation has begun decades back, it is still evolving with time. Building-Building interaction during emergency events with high speed communication network and effective control strategy, along with the existing energy optimization control loop will hold a brighter scope. With the intervention of wireless home automation, it can bind well with the emerging future technology, i.e. Internet Of Things (IOT), thus better control action can be envisaged.

\section{REFERENCES}

\section{Journal Papers}

1. Joao Figueiredo, Jose Sa da Costa, "A SCADA system for energy management in intelligent buildings", Energy and Buildings 2012, vol.49, pp 85-98

2. Kolokotsa D, Niachou K, Geros V, "Implementation of an integrated indoor environment and energy management system", Energy and Buildings 2009, vol.37(1), pp 93-99

3. Jerome Henry Kampf, Darren Robinson, "A simplified thermal model to support analysis of urban resource flows", Energy and Buildings vol.39, pp 445-453, 2011S. Joe Qin, Thomas A. Badgwell, "A survey of industrial model predictive control technology", Control Engineering Practice, 2003, Vol. 23, pp. 733-764

4. Kun Deng, PrabirBarooah, Prashant G. Mehta, "Building Thermal Model Reduction via Aggregation of States", Journal of Heat Transfer, 1999, vol.111(1), pp 5-12

5. X. Xu and S. Wang, "A simplified dynamic model for existing buildings using CTF and thermal network models," International Journal of Thermal Sciences, 2008, vol.47(9),pp 1249-1262

6. Lin, Yashen, Prabir, "Identification of control-oriented thermal models of rooms in multi-room buildings", IEEE Transaction on Control Systems Technology, 2009, vol.54(2), pp 31-39

7. YounghoonKwak, Jung-Ho $\mathrm{Hu}$, "Development of a model predictive control framework through real-time building energy management system data", Applied Energy, 2015, vol.155,pp 1-13

8. Giles Fraisse, Olivier Lafabrie, Gilbert Achard, Viardot, "Development of a simpliefied and accurate building model based on electrical and mechanical analogy", Energy and buildings, 2005, vol.34, pp 1017-1031

9. Kolokotsa, A Pouliezos, C Lazos, Stevi "Predictive control techniques for energy and indoor environmental quality management in buildings", Building and Environment, 2009, vol.44, pp 1850-1863

10. M.Gwerdr, B.Lehman, V.Dorer, "Control of Thermal Activated Building System (TABS)" Applied Energy, 2008, vol.85, pp 565-581

11. Vangelis Marinakis, HarisDoukas, CharikleiaKarakosta, John Psarras, "An integrated system for buildings' energy-efficient automation: Application in the tertiary sector", Applied Energy, 2013, vol.101, pp 6-14

12. Vangelis Marinakis, CharikleiaKarakosta, HarisDoukas, StylianiAndroulaki, John Psarras, "A building automation and control tool for remote and real time monitoring of energy consumption", Sustainable Cities and Society, 2013, vol. 41, pp 11-15

13 .

D.B.Crawley,J.W.Hand,M.Kummert,B.T.Griffith,Contra sting the capabilities of building energy performance simulation programs, 2008, Build.Environ.43, 661673.

ConferencePapers

14. Kummert M, Andre P., "Simulation of a model-based optimal controller for heating systems under realistic hypotheses", Proceedings of building simulation,9th conference of international building performance simulation association, 2005, pp 555-562 Book, book chapter and manual

15. Keith.J.Moss, "Energy Management in Buildings", 2nd Edition published by Taylor and Francis, UK, 2002

16. Shengwei Wang, "Intelligent Buildings and Building Automation", published by Spon press, New York, USA, 2010

17. J A Clarke, "Energy Simulation in Building Design", 2nd Edition published by Butterworth-Heinemann, 2001

18. Elena V.M. Papadopoulou, "Energy Management in Buildings using Photovoltaics, published by SpringerVerlag London Limited, 2012

Thesis

19. Michael Wetter, "Simulation Based Building Energy Optimization", $\mathrm{PhD}$ Thesis, University of California, Berkeley, 2004

Release Notes

20. "Advanced Energy Design Guide for Small Office Buildings", released by American Society of Heating, Refrigerating and Air-Conditioning Engineers (ASHRAE), North America, 2000

21. "Residential and Commercial Building energy survey", European Union, 2012. 